\section{On the deformation around holes described within a finite strain approach}

MARTIN LEDERER - Christian Doppler Laboratory for Lifetime and Reliability of Interfaces in Complex Multi-Material Electronics, TU Wien, Institute of Chemical Technologies and Analytics

GolTA KHATIBI - Christian Doppler Laboratory for Lifetime and Reliability of Interfaces in Complex Multi-Material Electronics, TU Wien, Institute of Chemical Technologies and Analytics Érkezett: 2017. 11. 01. - Received: 01. 11. 2017. - https://doi.org/10.14382/epitoanyag-jsbcm.2017.23
Martin LEDERER

Dr. Martin Lederer is researcher at a Christian Doppler Laboratory at TU Wien. He has received his Ph.D. from the University of Vienna, where he is still teaching as a lecturer.

Golta KHATIBI

Dr. Golta Khatibi is head of the Christian Doppler Laboratory RELAB at TU Wien. She has authored over 100 scientific publications.

\begin{abstract}
Numerous materials used in structural mechanics include a considerable amount of porosity. In order to assess their stability under load, it is necessary to evaluate the stress and strain concentrations around holes precisely. As a prerequisite for evaluations of stress distributions, an accurate definition of strain is required. For this purpose, we here propose logarithmic strain tensors in plane and spherical polar coordinates. It is demonstrated how the notion of incompressibility may accurately be described on the basis of a finite strain approach. Furthermore, the role of deviatoric and hydrostatic contributions to the stress is analyzed with respect to possible failure mechanisms.

Keywords: finite strain approach, material frame indifference, objectivity of a tensor, strain energy concentration

Kulcsszavak: véges nyúlások módszere, anyag nézôpont-függetlensége, tenzor objektivitás, alakváltozási energia koncentráció
\end{abstract}

\section{Introduction}

It is a well known fact that accurate calculations of structural mechanics should be done in the frame of a finite strain approach considering all the nonlinear effects, which are relevant for the stability of a structure. Nevertheless, the linearized theory of elastic deformations is still widely used, because evaluations may be carried out straightforwardly within reasonable time. Furthermore, the predictions of the linear theory are usually expected to converge against exact results in the limit of small deformations. However, the performance of advanced computer simulations has substantially increased in recent years. Therefore, the possible issue of excessive computation time related to nonlinear evaluations has lost its significance. Consequently, it is here suggested that calculations of structural mechanics should nowadays be performed on the basis of a nonlinear theory.

In this context, we here give an example, where the predictions of finite strain theory may be evaluated analytically. It is demonstrated how the condition of incompressibility is perfectly fulfilled during integration of the equilibrium condition. For this purpose, the logarithmic strain, also called Hencky strain $[1,2]$, is expressed in polar coordinates in order to evaluate axisymmetric problems. Thereby, the material volume of an incompressible solid is precisely preserved. In contrast, the linear theory can only approximate such a behavior, whereby large deformations are accompanied by increasing deviations from exact results.

In this context it should be mentioned that the linearized strain violates the principle of Euclidean objectivity, which represents a prerequisite for the principle of material frame indifference [3]. According to material objectivity, the response of a material should be independent of the position of an observer in space and time. But the linearized strain tensor does not fulfil the requirements of an objective tensor. Such considerations are already known in the theory of finite strains. However, these problems are seldom treated in plane or spherical polar coordinates. It is therefore the aim of the present study, to extend the principles of finite strain theory to geometries, which are naturally described in polar coordinates.

\section{Problem statement}

In order to demonstrate some issues related to the linearized strain tensor, the classical solutions for a hole under uniform stress are briefly addressed here. Let us assume a pipe consisting of an isotropic, linear elastic material under internal and external hydrostatic pressure, as depicted in Fig. 1.

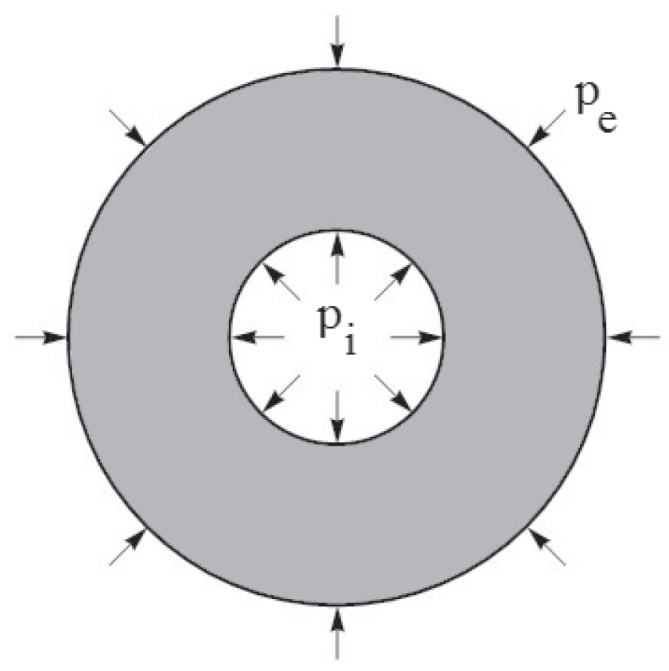

Fig. 1. A pipe under internal pressure $p_{i}$ and external pressure $p_{e}$, schematically 1. ábra Nyomás alatt álló csö sémája; belsö nyomás $p_{i}$ és külső nyomás $p_{e}$ 
In his historical publication, Lame [4] applied polar coordinates to solve this axisymmetric problem. In the axisymmetric case, the equilibrium condition for the stress $\sigma$ reads

$\frac{\partial \sigma_{r r}}{\partial r}+\frac{\sigma_{r r}-\sigma_{\theta \theta}}{r}=0$

where $r$ denotes the radial and $\theta$ the circumferential direction.

Further, axi-symmetry requires

$\tau_{r \theta}=0(2 \mathrm{a})$ and $\frac{\partial \sigma_{\theta \theta}}{\partial \theta}=0$

In this case, the linear strain tensor in polar coordinates writes as

$\varepsilon_{r r}=\frac{\partial u}{\partial r}, \varepsilon_{\theta \theta}=\frac{u}{r}$, and $\varepsilon_{r \theta}=0$.

Here, $u$ are the displacements in radial direction. On the basis of Hooke's law one arrives at the equation

$\frac{\partial^{2} u}{\partial r^{2}}+\frac{1}{r} \frac{\partial u}{\partial r}-\frac{u}{r^{2}}=0$

which is valid in both approximations, in plane strain as well as in plane stress. The general solution of this equation reads as

$u(r)=C_{1} \cdot r+\frac{C_{2}}{r}$

where $C_{1}$ and $C_{2}$ are coefficients which are to be determined from boundary conditions. In the limit of large values for the outer radius of the pipe, the sample represents a hole in an infinite plate.

Let us now carry out the cross-check whether conservation of volume is fulfilled, when the solution given above is applied to incompressible materials: In plane strain approximation, the condition for volume-conserving, axisymmetric deformations may be expressed as

$\varepsilon_{r r}+\varepsilon_{\theta \theta}=0$

Inserting the definitions of ( $3 \mathrm{a}, \mathrm{b})$ into (6) leads to the differential equation

$\frac{\partial u}{\partial r}+\frac{u}{r}=0$

This equation has the solution

$u(r)=\frac{C_{2}}{r}$

Consequently, the term including constant $\mathrm{C}_{1}$ may be omitted in equation (5) when incompressible solids are considered. One now may check the change of volume described by deformation of the form (6). Let the inner and outer radius of the undeformed state be $r_{i}$ and $r_{o}$, respectively. Assuming a normalized thickness of the plane strain model, the volume change $\Delta \mathrm{V}$ during deformation according to (8) is

$\Delta V=C_{2}^{2} \cdot \pi \cdot\left(\frac{1}{r_{o}}-\frac{1}{r_{i}}\right)$

It is true that this result approaches 0 in the limit of infinitesimal deformations. But unfortunately, the result of equation (9) is not exactly zero!

\section{Finite strain tensor in polar coordinates}

Finite strain tensors are well described in the Lagrangian representation of continuum mechanics. The relation between the coordinates $\mathrm{X}_{\mathrm{i}}$ of the deformed and the coordinates $\mathrm{x}_{\mathrm{i}}$ of the initial configuration is in Cartesian coordinates defined by the deformation gradient tensor

$F_{i j}=\frac{\partial X_{i}}{\partial x_{j}}$

Here, it should be mentioned that the exact condition of incompressibility can be expressed as

$\operatorname{det}(F)=1$.

From the deformation gradient, one obtains the CauchyGreen tensor $\mathrm{C}$ through

$C=F^{T} \cdot F$

where $\mathrm{F}^{\mathrm{T}}$ is the transposed matrix of $\mathrm{F}$. Thus, the stretch tensor $\mathrm{U}$ may be derived from the relation

$C=U^{2}$

The matrices $\mathrm{C}$ and $\mathrm{U}$ are both symmetric and have the same principal axes. Therefore, the stretch tensor $\mathrm{U}$ may also be expressed with the help of the outer product

$U=\sum_{a} \sqrt{\lambda_{a}} \cdot \vec{r}_{a} \otimes \vec{r}_{a}$,

where $\lambda_{\mathrm{a}}$ are the eigenvalues and $\vec{r}_{a}$ are the normalized eigenvectors of $\mathrm{C}$, respectively. Now, there are several possibilities how finite strain tensors $\mathrm{E}$ may be defined with use of $\mathrm{C}$ or $\mathrm{U}$. If one, however, likes to obtain a condition for incompressibility in the form of

$\operatorname{tr}(E)=0$,

where tr denotes the trace of a matrix, then the Hencky strain

$E=\ln (U)$

is the right choice. The Hencky strain is also called logarithmic strain. If one inserts (16) into (15), one gets a relation where the product of the sample dimensions remains constant, and this relation is equivalent to condition (11).

Let us now construct the equivalent expression in plane polar coordinates, whereby we here restrict ourselves to the axisymmetric case. Further, we here assume plane strain conditions. In consequence, we are left with two strain components pointing along orthogonal directions. By analogy to the Hencky strain one derives

$E_{r r}=\ln \left(1+\frac{\partial u}{\partial r}\right)$ and $E_{\theta \theta}=\ln \left(\frac{r+u}{r}\right)$

Moreover, a similar expression can be derived for spherical polar coordinates, where we have the coordinates $r, \theta$ and $\phi$. For simplicity, we focus our interest on the case of uniform multiaxial stress around a spherical hole. Due to symmetry reasons, the strain components $\mathrm{E}_{\mathrm{r} \theta}, \mathrm{E}_{\mathrm{r} \phi}$ and $\mathrm{E}_{\mathrm{q} \phi}$ are zero. Thus, one obtains

$E_{r r}=\ln \left(1+\frac{\partial u}{\partial r}\right), E_{\theta \theta}=E_{\varphi \varphi}=\ln \left(\frac{r+u}{r}\right)$. 
Using the here mentioned strain tensors in plane or spherical polar coordinates together with the required symmetry conditions, one can express incompressibility of a material according to equation (15).

\section{Integration of differential equations}

We are now in the position to integrate the differential equations, which are obtained by inserting the logarithmic strain in polar coordinates into the incompressibility condition. In the case of plane strain, inserting of (17) into (15) yields

$1+\frac{\partial u}{\partial r}=\frac{r}{u+r}$

after simplification. The solution of this differential equation reads as

$u(r)=\sqrt{r^{2}+a}-r$

where the integration constant a is related to the amount of deformation. It is easily verified that a displacement field described by (20) preserves the volume of a body during deformation. Furthermore, a numerical similarity between the solutions (8) and (20) can be found for small values of a.

By analogy, the three-dimensional case of equation (18) may be evaluated. This time, one obtains the differential equation

$1+\frac{\partial u}{\partial r}=\left(\frac{r}{u+r}\right)^{2}$.

The solution of this equation reads as

$u(r)=\sqrt[3]{r^{3}+b}-r$,

where $b$ denotes the integration constant. Like in the previous case, conservation of volume may easily be verified for this deformation mode.

It should be noticed that the solutions (20) and (22) are not restricted to purely elastic materials. Instead, they may also be applied to plastic deformations, but a detailed material model would be necessary to evaluate further details. Furthermore, the precise treatment of strain is also relevant for materials undergoing a volume change under hydrostatic pressure. In fact, many construction materials show a dependence of their strength on hydrostatic pressure, as for instance considered in the Drucker-Prager model [5] or its novel modifications [6]. In this context, the precise use of strain tensors is a prerequisite for implementation of such material models in computer simulations.

\section{Solutions for elastic materials}

Here, the solutions for isotropic, linear elastic materials are elaborated. Henceforth, compressible and incompressible materials are both included in the considerations. Further, we here at first consider the geometry and the loading case depicted in Fig. 1.

In view of the axisymmetric geometry investigated here, it appears convenient to rewrite the strain tensor
$E=\left(\begin{array}{ccc}E_{r r} & 0 & 0 \\ 0 & E_{\theta \theta} & 0 \\ 0 & 0 & 0\end{array}\right)$

in terms of deviatoric and hydrostatic strains:

$E^{d e v}=E-\frac{1}{3} \operatorname{tr}(E) \cdot I$

and

$E^{\text {hyd }}=\frac{1}{3} \operatorname{tr}(E) \cdot I$,

where I is the 3-dimensional identity matrix. The corresponding deviatoric and hydrostatic stresses are:

$\sigma^{d e v}=\sigma-\frac{1}{3} \operatorname{tr}(\sigma)$

$\sigma^{\text {hyd }}=\frac{1}{3} \operatorname{tr}(\sigma) \cdot I$

Per definition, the hydrostatic stress tensor is related to the pressure

$p=-\frac{1}{3} \operatorname{tr}(\sigma)$

in the material. The orthogonal decomposition of stresses and strains permits the separation of material properties related to compression and shear:

For isotropic materials, two independent material parameters are needed. We use the bulk modulus $\mathrm{K}$ related to the volume change and the shear modulus $G$ describing deviatoric deformations. When now Hooke's law is reformulated with use of the logarithmic strain, it is necessary to avoid a change of the material definitions. Hence, logarithmic strain components must, for the moment, be converted to their linearized amounts. This step is achieved according to the rules converting true strains $\mathrm{E}$ into linearized strains $\varepsilon$. Since

$E=\ln (1+\varepsilon)$,

the inverse transformation writes

$\varepsilon=\exp \{E\}-1$.

Of course, it would also be possible to utilize some hyperelastic material law instead of keeping the definitions of a Hookean material. However, it is the purpose of the present investigation to study the effect of an improved strain definition without making changes to the material law.

Consequently, Hooke's law may be rewritten in the simple form

$\sigma^{d e v}=2 G \cdot\left(\exp \left\{E^{d e v}\right\}-I\right)$,

$p=-K \cdot(\exp \{\operatorname{tr}(E)\}-1)$.

In the context of finite strain theory, deviatoric and hydrostatic contributions to displacements may be considered in consecutive steps. The hydrostatic deformation mode is simply of the form $u=C_{1} \cdot r$. Inserting the coordinates of the hydrostatically deformed state as initial conditions into equation (20) yields 


$$
u(r)=\sqrt{(c+1)^{2} \cdot r^{2}+a}-r,
$$

where the constants $c$ and a are to be determined from the boundary conditions in combination with the material law. Here, it should be recognized that the intermediate transformation into a linearized strain according to $(27 \mathrm{~b})$ did not disturb the improvements achieved by use of the logarithmic strain.

By combination of the equations (23) through (29), the hydrostatic pressure inside the elastic material turns out to be

$$
p=-K \cdot\left(2 c+c^{2}\right)
$$

We are now in the position to solve the boundary value problem

$\sigma_{r r}\left(r_{i}\right)=-p_{i}$

and

$\sigma_{r r}\left(r_{o}\right)=p_{e}$

where $\mathrm{p}_{\mathrm{i}}$ and $\mathrm{p}_{\mathrm{e}}$ stand for the gas pressure at the inside and the outside of the pipe. One obtains the equations

$-p_{i}=2 G\left(\frac{\sqrt[3]{c+1}^{2} \cdot r_{i}}{\sqrt{(c+1)^{2} r_{i}^{2}+a}}-1\right)+K\left(2 c+c^{2}\right)$

and

$p_{e}=2 G\left(\frac{\sqrt[3]{c+1}^{2} \cdot r_{o}}{\sqrt{(c+1)^{2} r_{o}^{2}+a}}-1\right)+K\left(2 c+c^{2}\right)(32 \mathrm{a}, \mathrm{b})$

for the constants $\mathrm{c}$ and $\mathrm{a}$.

By analogy, one may also derive the solution for a spherical hole described by the strain tensor (18 a-c). Deviatoric and hydrostatic contributions to the strain take again the general form of equations (24 a, b). The same can be said about equations (25) through (28). The displacement field related to the spherical hole under hydrostatic pressure is

$u(r)=\sqrt[3]{(c+1)^{3} \cdot r^{3}+b}-r$

for isotropic elastic materials. For this geometry, the hydrostatic pressure inside the material reads as

$p=-K\left(c^{3}+3 c^{2}+3 c\right)$

The boundary value problem may again be formulated in the style of equation ( $31 \mathrm{a}, \mathrm{b})$. Consequently, one derives

$$
\begin{gathered}
-p_{i}=2 G\left(\frac{(c+1)^{2} \cdot r_{i}^{2}}{{\sqrt[3]{(c+1)^{3} r_{i}^{3}+b}}^{2}}-1\right)+ \\
K\left(c^{3}+3 c^{2}+3 c\right)
\end{gathered}
$$

and

$$
\begin{gathered}
p_{e}=2 G\left(\frac{(c+1)^{2} \cdot r_{o}^{2}}{{\sqrt[3]{(c+1)^{3} r_{i}^{3}+b}}^{2}}-1\right)+ \\
K\left(c^{3}+3 c^{2}+3 c\right) .
\end{gathered}
$$

The values for the constants a and c may be determined from this system of equations.

\section{Concentration of strains around holes}

Usually, the effect of the stress on a defect is discussed in terms of stress concentration factors. However, it has already been suggested to focus the interest rather on the increase of strain energy around a defect [7]. Indeed, strain energy concentration factors are not identical with stress concentration factors. The strain concentration strongly depends on the relation between the material parameters $\mathrm{K}$ and $\mathrm{G}$. We will hereafter show that extremely high strain energy concentrations are found around holes in nearly incompressible materials. For this purpose, the orthogonal decomposition of stresses and strains into deviatoric and hydrostatic parts is once again utilized. Within the linearized approximation the strain energy density reads as $w=\frac{1}{2} \sigma: \varepsilon=\frac{1}{2} K \cdot \operatorname{tr}(\varepsilon)^{2}+G \cdot \varepsilon^{d e v}: \varepsilon^{d e v}$

In the finite strain approach, it is necessary to consider the volume change aside from equations $(28 \mathrm{a}, \mathrm{b})$. Thus, one obtains

$W=W^{h y d}+W^{d e v}$

with

$$
\begin{aligned}
& W^{\text {hyd }}=\int_{V} \frac{K}{2}\left(\exp \{\operatorname{tr}(E)-1\}^{2}\right) d V \\
& W^{\text {dev }}=\int_{V} G\left(\exp \left\{E^{d e v}\right\}-I\right)^{2} \cdot \exp \{\operatorname{tr}(E)\} d V
\end{aligned}
$$

Let us now assume the geometry of a hole in an infinite plate under uniform biaxial tension in plane strain approximation. This means, we consider the geometry of Fig. 1 in the limit of $r_{o} \rightarrow \infty$. At the hole, the boundary condition writes as

$\sigma_{r r}=0$.

In order to solve this boundary value problem, it should be recognized that the hydrostatic pressure is constant across the whole sample volume due to equation (30). Further, the radial components of hydrostatic and deviatoric stress tensor must be on balance at the inside of the hole. This means, the correlated radial components of equations ( $25 \mathrm{a}$ and $\mathrm{b}$ ) must compensate there:

$\sigma_{r r}-\frac{1}{3}\left(\sigma_{r r}+\sigma_{\theta \theta}+\sigma_{z z}\right)=p$

Considering the boundary condition (38) and $\sigma_{z z}=-p$, one derives

$\sigma_{\theta \theta}=2 p$

for the circumferential stress at the hole. In combination with equation (38) this leads to a stress concentration factor of $\mathrm{K}_{\mathrm{t}}=$ 2 at the hole. On the other hand, the related deviatoric strain component at the hole may be written as

$E_{r r}^{d e v}=E_{r r}-\frac{1}{3} \operatorname{tr}(E)$

Consequently, the equation

$\sigma_{r r}^{d e v}=2 G \cdot\left(\exp \left\{E_{r r}^{d e v}\right\}-1\right)$ 
holds. Let us now compare the strain $E_{r r}(\infty)$ far away from the hole with the strain $E_{r r}^{d e v}\left(r_{i}\right)$ at the hole. Thus, one obtains

$\frac{E_{r r}^{d e v}\left(r_{i}\right)}{E_{r r}(\infty)}=\frac{\ln \left(1-\frac{p}{2 G}\right)}{\frac{1}{2} \ln \left(1-\frac{p}{K}\right)}$

In the limit for small deformations one obtains

$\frac{E_{r r}^{d e v}\left(r_{i}\right)}{E_{r r}(\infty)} \rightarrow \frac{K}{G}$.

Now, it has become clear that the strain concentration factor depends on the ratio $\mathrm{K} / \mathrm{G}$ of the material properties. Consequently, the strain energy (37) includes a term of this type.

The evaluations for the spherical hole can be derived by analogy. For nearly incompressible materials, extremely high concentrations of strain energy are found at the hole. For large strains, the correction of finite strain theory is governed by the relation between linear and logarithmic strains.

\section{Discussion}

The definition of strain is in principle independent of the materials described. However, a precise definition of material properties requires that the principle of material frame indifference is satisfied. An objective strain tensor must include an accurate description of the volume change in the material. In this respect, the linearized strain tensor leads to ambiguities, because it violates the principle of Euclidean objectivity. We have therefore used an objective strain tensor, which applies to axisymmetric problems. For this purpose, the logarithmic strain was formulated for polar coordinates. In this way, the material response to hydrostatic pressure could be described precisely. A separation of deviatoric and hydrostatic deformation modes was achieved on the basis of an orthogonal decomposition of stresses and strains. In conclusion, the concentrations of strain energy near holes could be evaluated.

Usually, evaluation methods derived from the linearized theory are justified in the sense that solutions are easy to calculate. In many applications, however, the solutions of structural mechanics are evaluated on numerical level. Since the efficiency of computer systems has enormously improved in recent years, the argument suggesting that the linearized strain was easy to compute has lost its relevance. In context with computer simulations, it is often of advantage to work with precise mathematical definitions, because of better convergence behavior observed for iterative algorithms.

Nevertheless, it must be said that in the limit of infinitesimal small deformations, the results of the present study agree with predictions of linear elasticity.

\section{Summary and conclusions}

The deformations around holes in isotropic elastic materials were evaluated in the frame of finite strain theory. The logarithmic strain tensor was applied to plane and to spherical polar coordinates, whereby axisymmetric geometries were considered. Owing to the symmetry of the problem, the equilibrium conditions simplified to ordinary differential equations, which could be solved straightforwardly. In consequence, the principle of material frame indifference was strictly fulfilled.

An interesting detail consistsin the orthogonal decomposition of stresses and strains into deviatoric and hydrostatic parts. In this way the material properties related to shear modulus $G$ and compression modulus $\mathrm{K}$ may be examined separately. In particular, a low value of the shear modulus $\mathrm{G}$ in comparison to the bulk modulus $\mathrm{K}$ can lead to tremendously high values of the strain energy due to large deviatoric strains near the hole.

The geometries considered here are very simple. On the other hand, analytical exact solutions are usually not derived for complex structures. Nevertheless, the methods developed here point the way how similar results can be obtained for structures of general shape. Moreover, the geometry of small holes inside a structure is of high relevance for materials with porosity. Insofar, our results apply to numerous materials used for structural mechanics.

\section{Acknowledgements}

The financial support of the Austrian Ministry of Science, Research and Economy and the National Foundation for Research, Technology and Development is gratefully acknowledged.

\section{References}

[1] H. Hencky (1925): Die Bewegungsgleichungen beim nicht-stationären Fließen plastischer Massen, Zeitschrift f. technische Physik, Vol. 9, pp. 215220.

[2] H. Hencky (1929): Über die Form des Elastizitätsgesetzes bei ideal elastischen Stoffen, Journal of Rheology, Vol. 2, pp. 169-176.

[3] I-S. Liu (2004): On Euclidean objectivity and the principle of material frame-indifference, Continuum Mechanics and Thermodynamics, Vol. 16, pp. 177-183. https://doi.org/10.1007/s00161-003-0149-x

[4] G. Lame (1852): Lecons sur la theorie mathematque de l' elasticite, Paris: Bachelier

[5] D. C. Drucker - W. Prager (1952): Soil mechanics and plastic analysis or limit design. Quarterly of Applied Mathematics, Vol. 10, No. 2, pp. 157165.

[6] L. H. Han - J. A. Elliott - A. C. Bentham - A. Mills - G. E. Amidon B. C. Hancock (2008): A modified Drucker-Prager Cap model for die compaction simulation of pharmaceutical powders. International Journal of Solids and Structures, Vol. 45, pp. 3088-3106. https://doi.org/10.1016/j.ijsolstr.2008.01.024

[7] Z. Yang (2009): Stress and strain concentration factors for tension bars of circular cross-section with semicircular groove. Engineering Fracture Mechanics, Vol. 76, pp. 1683-1690. https://doi.org/10.1016/j.engfracmech.2009.03.005

$\underline{\text { Ref.: }}$

Lederer, Martin - Khatibi, Golta: On the deformation around holes described within a finite strain approach Építőanyag - Journal of Silicate Based and Composite Materials, Vol. 69, No. 3 (2017), 127-131. p. https://doi.org/10.14382/epitoanyag-jsbcm.2017.23 